\title{
Repeated Treatment Protocols for Melasma and Acquired Dermal Melanocytosis
}

\author{
Kotaro Yoshimura, MD, Katsujiro Sato, MD, Emiko Aiba-Kojima, MD, \\ Daisuke Matsumoto, MD, Chiaki Machino, MD, Takashi Nagase, MD, \\ Koichi Gonda, MD, and Isao Koshima, MD*
}

BACKGROUND AND OBJECTIVE Melasma and acquired dermal melanocytosis (ADM; acquired bilateral nevus of Ota-like macules) are both seen most commonly symmetrically on the face of women with darker skin and are also known as difficult conditions to treat.

METHODS Our topical bleaching protocol with 0.1 to $0.4 \%$ tretinoin gel and $5 \%$ hydroquinone was performed repeatedly (1-3 times) for melasma ( $n=163$ ), and a combination treatment with topical bleaching and Q-switched ruby (QSR) laser was performed repeatedly (1-3 times) for ADM $(n=62)$.

RESULTS There is a significant correlation between clinical results (clearance of pigmentation) and the number of sessions in both melasma $(p=.019)$ and $\operatorname{ADM~}(p<.0001)$.

CONCLUSION The repeated treatment protocol for melasma and ADM showed successful clinical results compared with conventional ones, and they may be applied to other pigment conditions. It may be better that epidermal and dermal pigmentations are treated separately, especially in dark-skinned people who are more likely to suffer postinflammatory hyperpigmentation after inflammation-inducing therapies.

The authors have indicated no significant interest with commercial supporters.

$\mathrm{M}$ elasma is acquired and symmetrical hypermelanosis, usually spread widely on the malar prominence and cheek, and less frequently on the forehead and upper lip. Melasma usually appears in patients in their 30 s or 40 s after pregnancy or contraceptive use, suggesting that the triggering of melasma is hormonally related. ${ }^{1}$ Conventional treatments for melasma include sunscreen, hypopigmenting agents, often in combination with other therapies, such as tretinoin, topical corticosteroids, or superficial peeling agents. ${ }^{2-7}$ On the other hand, acquired dermal me- lanocytosis $(\mathrm{ADM})$ is a pigmented lesion involving bilateral grayishbrown facial macules first reported as acquired bilateral nevus of Ota-like macules (Hori's nevus) by Hori and colleagues ${ }^{8} \mathrm{ADM}$ usually onsets in patients in their 20 s and represents bilateral involvements, with the malar regions almost always affected while the lateral forehead and nasal alas are sometimes involved. The distribution pattern, its grayish round-spot appearance with unclear margins, and the difference in color are critical points that distinguish it from melasma. As both melasma and ADM are bilateral lesions and some patients have both, inexperienced doctors could misdiagnose them.

Melasma and ADM are frequently seen in Oriental females, and indeed 225 of 1,184 patients $(19.1 \%)$ who were treated for pigmented skin problems in our outpatient clinic had either or both. The authors previously described an aggressive and optimal use of tretinoin along with hydroquinone for various kinds of skin hyperpigmentation ${ }^{9-11}$ and a combination therapy with Qswitched ruby (QSR) laser for ADM. ${ }^{12}$ The topical bleaching

*All authors are affiliated with Department of Plastic Surgery, University of Tokyo, School of Medicine, Tokyo, Japan 
treatment with tretinoin and hydroquinone is a most effective tool for removal of epidermal pigmentation. In this study, the clinical results of repeated therapies for melasma and ADM were analyzed; we performed repeated tretinoin-hydroquinone bleaching therapy for melasma, and a repeated combination therapy of topical bleaching and QSR laser for ADM.

\section{Patients and Methods}

\section{Preparation of Ointments}

Tretinoin aqueous gels (tretinoin gel) at three different concentrations $(0.1 \%, 0.2 \%$, and $0.4 \%)$, an ointment including $5 \%$ hydroquinone and 7\% lactic acid (HQ-LA ointment), and one including 5\% hydroquinone and $7 \%$ ascorbic acid (HQ-AA ointment) were originally prepared at the Department of Pharmacy, University of Tokyo Hospital. The precise regimens of these ointments have been described before. ${ }^{10,12}$ These gels can be prepared relatively easily because the tretinoin powder (Sigma Chemical, St. Louis, MO, USA) is commercially available. Aqueous gel is most suitable for the ointment base of tretinoin because of its good permeability. Tretinoin gel is pharmacologically unstable, so fresh batches were prepared at least once a month and stored in a dark, cool $\left(4^{\circ} \mathrm{C}\right)$ place.

\section{Evaluations of Results}

Photographs were taken for every patient at baseline and after treatment with a high-resolution digital camera (EOS-D30, Canon, Tokyo, Japan). The percentage of pigmentary clearance was evaluated via photographs by two experienced plastic surgeons who did not perform this treatment. The mean data of the pigmentary clearance of each patient were classified into four categories: "excellent" $(80 \%$ or more clearance), "good" (50\% to less than $80 \%$ clearance), "fair" ( $0 \%$ to less than $50 \%$ clearance), and "poor" (no change or worse).

\section{Patients}

Of 1,184 Asian patients who underwent cosmetic treatments, 163 had melasma and 62 suffered from ADM (six also had melasma). All patients with melasma or ADM were women except for two men with melasma. Patient age at the start of the treatment for melasma and ADM ranged from 27 to 62 years $(42.3 \pm 7.1$; mean \pm SD) and from 22 to 53 years $(36.4 \pm 8.1)$, respectively.
Sites affected by melasma or ADM are summarized in Table 1.

\section{Treatment Methods}

For melasma, our topical bleaching treatment was performed. If patients wanted, the treatment was repeated two or three times. For ADM, a combination therapy of topical bleaching and QSR laser was performed. The number of treatment sessions depended on the patient's decision. Typical time courses of the treatment protocols are shown in Figure 1A and B.

\section{(1) Topical bleaching treatment:}

The purpose of this treatment is to improve epidermal pigmentation by accelerating discharge of epidermal melanin (with tretinoin) and suppressing new epidermal melanogenesis (with hydroquinone). The two-stage (bleaching and healing) treatment was performed as follows:

(a) Bleaching phase: $0.1 \%$ tretinoin gel and HQ-LA ointment were initially applied to the
TABLE 1. Summary of Frequency of Melasma or Acquired Dermal Melanocytosis (ADM) Affected Sites in Patients

\begin{tabular}{lcc} 
& Melasma & ADM \\
\hline Forehead & $10(6.1 \%)$ & $22(35.5 \%)$ \\
Upper eyelids & $43(26.4 \%)$ & $10(16.1 \%)$ \\
Lower eyelids & & $15(24.2 \%)$ \\
Nasojugal groove & $18(29.0 \%)$ \\
Malar prominence & $157(96.3 \%)$ & $53(85.5 \%)$ \\
Cheek & $90(55.2 \%)$ & $14(22.6 \%)$ \\
Nasal dorsum & $68(41.7 \%)$ & $9(14.5 \%)$ \\
Nasal ala & $38(23.3 \%)$ & \\
Upper lip & $16(9.8 \%)$ & 141 sites \\
Lower lip & 422 sites & 62 cases \\
& 163 cases & \\
\hline
\end{tabular}


A

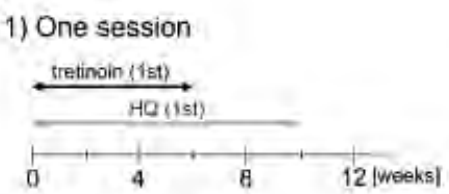

2) Two sessions

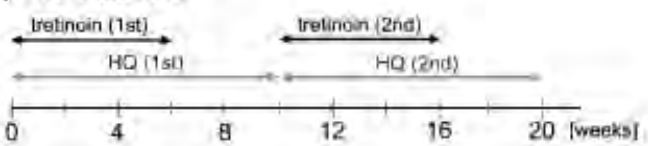

3) Three sessions

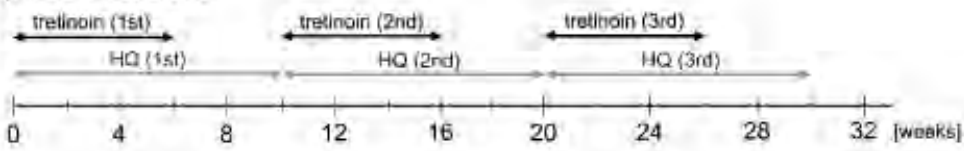

B

1) One session

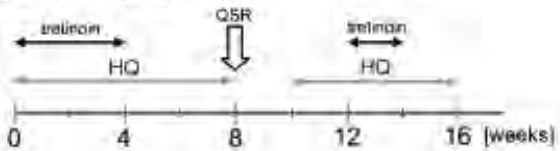

2) Two sessions

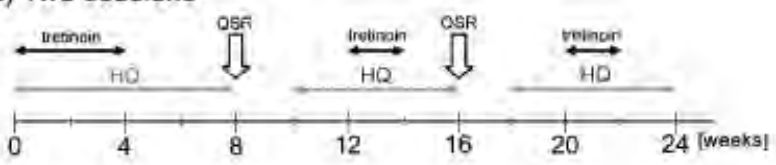

3) Three sessions

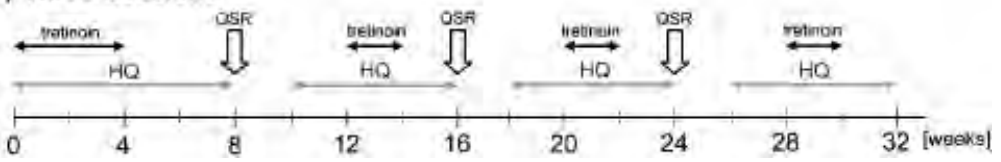

Figure 1. (A) A representative time course of our topical bleaching treatment with tretinoin and hydroquinone. Tretinoin is used for 6 weeks in each bleaching phase, and can be restarted with at least an 4-week interval of healing phase. (B) A representative time course of the combined treatment. Tretinoin is used for 4 weeks in the initial bleaching pretreatment, and for 2 weeks in the following pretreatments. If $\mathrm{Q}$-switched ruby (QSR) laser treatment is performed three times, the total treatment period is 32 weeks.

skin lesions twice a day. A small amount of tretinoin gel was carefully applied only on pigmented spots using a small cotton-tip applicator (an excessive volume of tretinoin gel can be wiped off), while the HQ-LA ointment was widely applied with fingers (eg, all over the face) a few minutes later, after allowing the ap- plied tretinoin aqueous gel to dry. The method of ointment application is critical in this aggressive treatment in order to obtain maximal bleaching effects with minimal irritant dermatitis. In cases in which severe irritant dermatitis was induced by the HQ-LA ointment, HQ-AA ointment was used instead. Patients were re- quested to visit our hospital at $1,2,4,6$, and 8 weeks after starting this treatment, and every 4 weeks thereafter. When the appropriate skin reaction (mild erythema and scaling) was not observed at 1 week, the concentration of tretinoin was increased to $0.4 \%$, because $0.2 \%$ tretinoin gel was usually not strong enough to get a sufficient reaction in these cases. The concentration of tretinoin and frequency of its application were appropriately modified according to the skin condition and degree of erythema and scaling. It took 4 to 8 weeks to finish this phase. In the second or third bleaching treatment, tretinoin gel of the final strength used in the most recent step was used from the beginning.

(b) Healing phase: After a 4- to 8week bleaching phase, the application of tretinoin gel and HQ-LA ointment was discontinued, and application of HQAA ointment was started in order to prevent postinflammatory hyperpigmentation (PIH) until the redness was sufficiently reduced. It usually took 4 weeks to complete this phase. Topical corticosteroids were not used in either the bleaching or healing phase.

(2) QSR laser treatment: In patients with ADM, topical anesthesia (lidocaine patch; Penles ${ }^{\circledR}$, Wyeth Lederle Japan Inc., Tokyo, Japan) was applied 60 to 120 
TABLE 2. Clinical Results of Cases with Melasma

\begin{tabular}{lccccccc} 
& Excellent & Good & Fair & Poor & Total & $\begin{array}{l}\text { Excellent or } \\
\text { Good Cases (\%) }\end{array}$ & $\begin{array}{l}\text { Excellent } \\
\text { Cases (\%) }\end{array}$ \\
\hline One treatment & & & & & & & \\
Two treatments & 25 & 40 & 27 & 4 & 96 & 67.7 & 26.0 \\
Three treatments & 7 & 20 & 15 & 0 & 56 & 73.2 & 37.5 \\
Total & 53 & 3 & 1 & 0 & 11 & 90.9 & 63.6 \\
\hline
\end{tabular}

*The treatment means a topical bleaching treatment (See Figure 1A).

minutes before the laser treatment. For QSR 694.5 nm laser (Model IB101, Niic Co. Ltd., Tokyo, Japan) treatment, $5 \mathrm{~mm}$ spot size, $1 \mathrm{~Hz}$ repeat rate, $20 \mathrm{~ns}$ pulse duration, and 4.0 to $5.0 \mathrm{~J} / \mathrm{m}^{2}$ fluences were used. After laser treatment, topical gentamicin sulfate ointment (Gentacin ${ }^{\circledR}$, Schering-Plough, NJ, USA) was applied twice a day until a scale or thin crust disappeared (usually 57 days). At 2 weeks after laser treatment, application of HQ-AA ointment was started.

At 4 weeks after each laser treatment, topical bleaching treatment with tretinoin gel of appropriate concentration (usually the same as the final concentration in the bleaching phase) and HQ-AA ointment were started as a pretreatment for the next laser irradiation, and also as a treatment for postlaser PIH in some cases. In most cases, a bleaching phase for 2 weeks was sufficient, and we can usually estimate the clinical results at 8 weeks after each laser treatment. When some hyperpigmentation remains, we can carry out another session.

\section{Statistics}

Spearman's correlation coefficient by rank test was used to analyze statistical significance between the extent of clinical improvement and the number of treatments.

\section{Results}

In 163 patients with melasma, 96 underwent only one topical bleaching treatment (see Figure 1A) with excellent results in 25 and good in 40; 56 underwent two treatments with excellent results in 21 and good in 20; 11 underwent three treatments with excellent results in 7 and good in
3 (Table 2). In 62 patients with ADM, 16 underwent only one treatment (a combination of topical bleaching and QSR laser; see Figure 1B) with excellent results in 1 and good in 6; 26 underwent two treatments with excellent results in 14 and good in 8; and 20 underwent three treatments with excellent results in 17 and good in 3 (Table 3). Representative cases with melasma are shown in Figures 2 and 3, and those with $\mathrm{ADM}$ in Figures 4 and 5.

As for side effects, mild erythema and scaling are indications of appropriate administration of tretinoin and were seen in almost all cases during the bleaching phase. Patients were well informed about the local adverse effects in advance and were requested to apply the tretinoin gel on only highly pigmented areas to keep the dermatitis as limited as possible. PIH

TABLE 3. Clinical Results of Cases with Acquired Dermal Melanocytosis (ADM)

\begin{tabular}{lccccccc} 
& Excellent & Good & Fair & Poor & Total & $\begin{array}{l}\text { Excellent or } \\
\text { Good Cases (\%) }\end{array}$ & $\begin{array}{l}\text { Excellent } \\
\text { Cases (\%) }\end{array}$ \\
\hline One treatment* & 1 & 6 & 9 & 0 & 16 & 43.8 & 6.3 \\
Two treatments & 14 & 8 & 4 & 0 & 26 & 84.6 & 53.8 \\
Three treatments & 17 & 3 & 0 & 0 & 20 & 100.0 & 85.0 \\
Total & 32 & 17 & 13 & 0 & 62 & 79.0 & 51.6 \\
\hline
\end{tabular}

*The treatment means a combination of topical bleaching treatment and Q-switched ruby laser (See Figure 1B). 

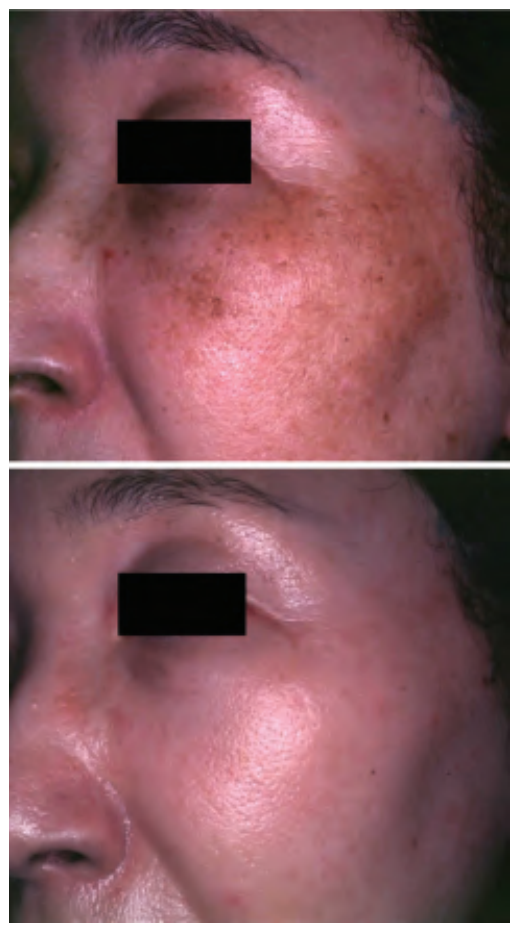

Figure 2. Case 1. (top) Baseline photo of a 47-year-old woman with melasma. (bottom) At 5 months, the pigmentation was cleared up after three sessions of topical bleaching treatments.

was seen after the first session of the repeated treatments in $14 \%$ of melasma patients, and a few weeks after the first QSR irradiation in $21 \%$ of $\mathrm{ADM}$ patients. The PIH was easily treated with the following topical bleaching treatment in both melasma and ADM patients.

Statistical analysis showed there is a significant correlation between clinical results (clearance of pigmentation) and the number of sessions in both melasma $(p=.019)$ and $\operatorname{ADM}(p<.0001)$.

\section{Discussion}

The authors previously reported on a topical bleaching therapy

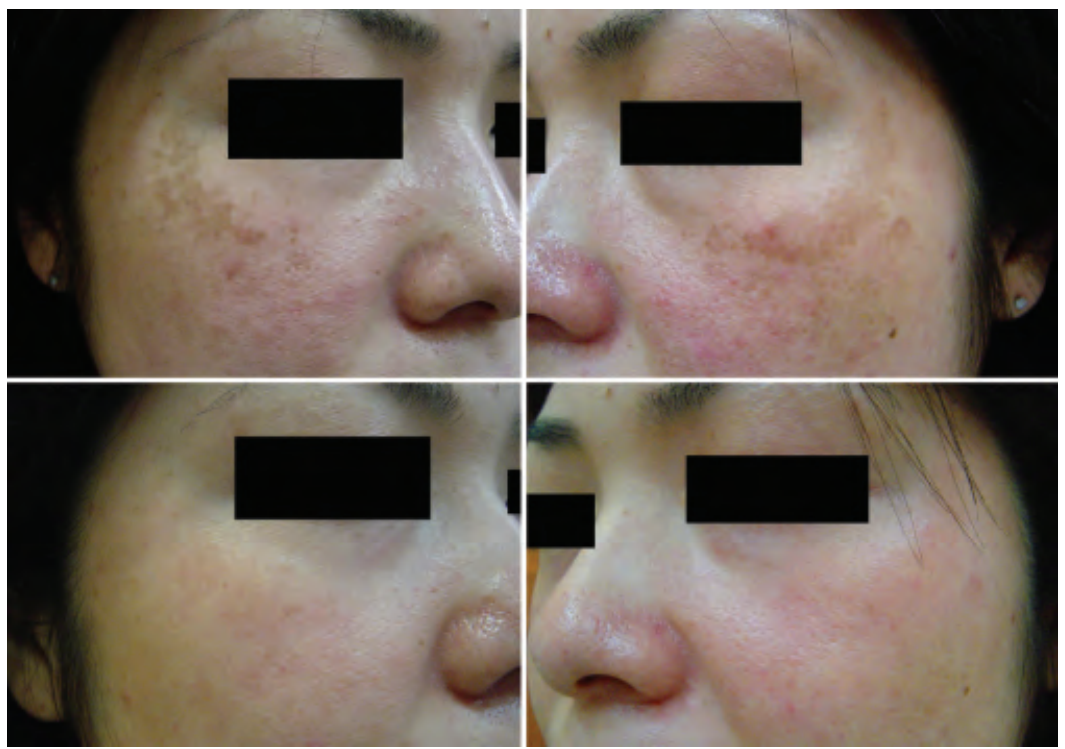

Figure 3. Case 2. (top, left, and right) A baseline view of a 27-year-old woman with melasma. (bottom, left, and right) At 5 months, after two sessions of Q-switched ruby laser and topical treatments. The clinical result was evaluated as "excellent."
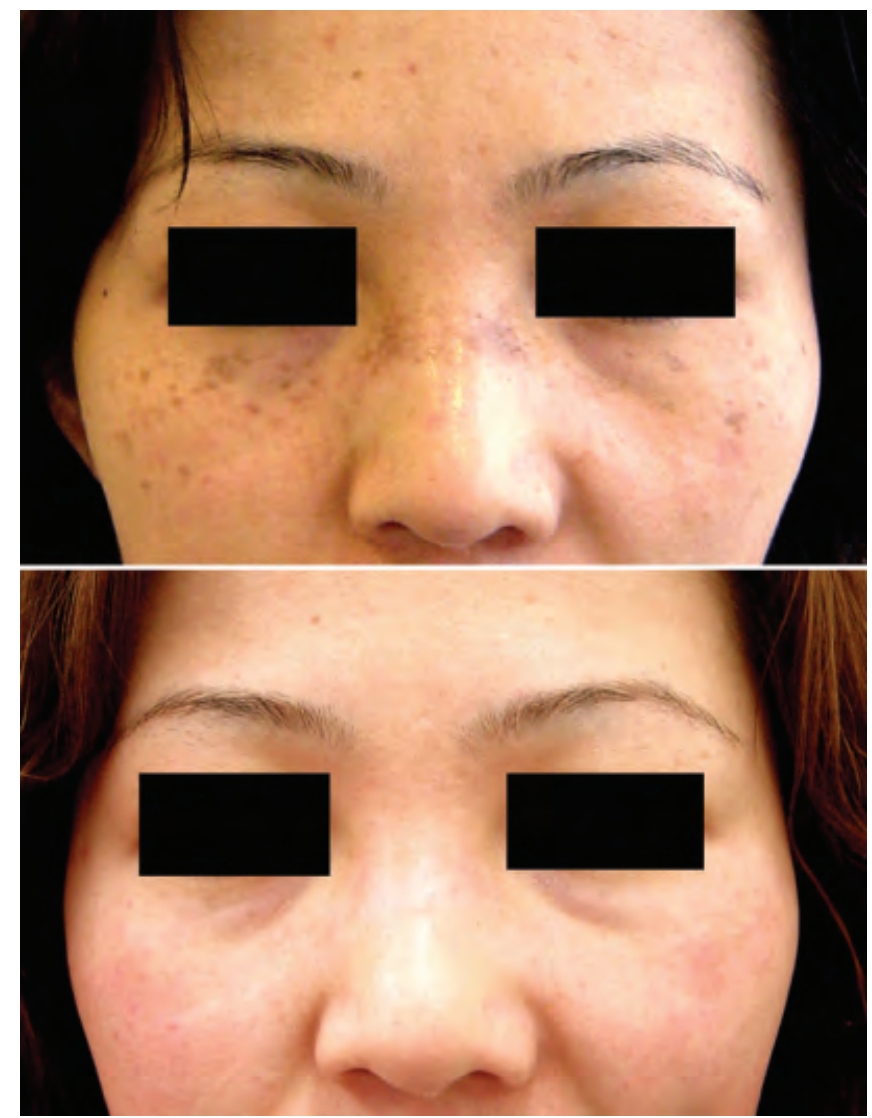

Figure 4. Case 3. (top) A baseline view of a 41-year-old woman with acquired dermal melanocytosis. (bottom) Two months after the third Q-switched ruby laser treatment $(8$ months from the baseline). The result of the clearance was evaluated as "excellent." 

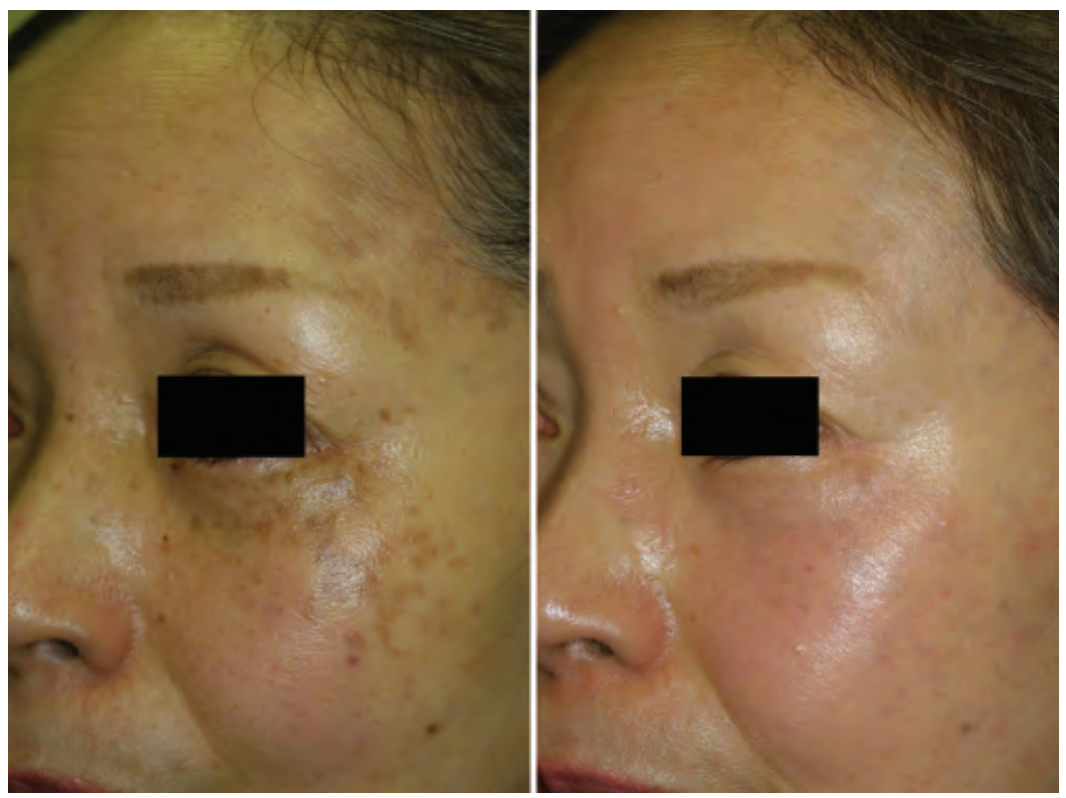

Figure 5. Case 4. (left) A baseline view of a 49-year-old woman with acquired dermal melanocytosis. She had spotty pigmentations on the cheeks, lateral forehead, and nasal alars. (right) After three sessions of Q-switched ruby laser and topical treatments ( 32 weeks from the baseline). The pigmentations were almost completely cleared and also the yellowish color of surrounding skin changed to pinkish.

with aggressive use of retinoids in aqueous gel only on the pigmented spots and use of hydroquinone all over the face. ${ }^{12,13}$ This treatment can only clear epidermal pigmentation, but with excellent efficiency compared with other conventional treatments, such as AHA peeling or single applications of tretinoin or hydroquinone. Corticosteroids are not used in the bleaching protocol, and, furthermore, tretinoin is not continually used over 2 months. It is well known that long-term continual use of tretinoin, either topical or oral, reduces its clinical effects. ${ }^{14,15}$ It has been suggested that this phenomenon may be due to intracellular production of cellular retinoic acid binding proteins (CRABPs) induced by the retinoid signal. This is why we use repeated bleaching protocols with intervals instead of continual tretinoin use.

The present results demonstrate that the results for ADM (excellent cases; excellent and good cases $=6.3 \% ; 43.8 \%)$ are not as good as for melasma (26.0\%; $67.7 \%$ ) in one-session cases, but in three-session cases ADM was improved at a higher rate $(85.0 \%$; $100 \%)$ than melasma $(63.6 \%$; $90.9 \%$ ). Actually, we often detected apparent improvement during the second session in cases with ADM.

Melasma usually has most of its pigmentation in the epidermis. Although previous reports with tretinoin, hydroquinone, AHA or others, or combinations of multiple agents showed moderate to relatively good clearance of melasma, ${ }^{2-7}$ complete clearance of pigmentation is rare. On the basis of our experiences, the differential use of tretinoin and hydroquinone is quite important for melasma, because if we use tretinoin on a larger area such as the whole face, the surrounding nonpigmented area is also bleached, and consequently the macules would remain clinically recognizable. Although melasma is well known as a difficult condition to treat, repetition of the topical bleaching on only the pigmented area improved it completely in some cases.

ADM has significant epidermal pigmentation, unlike nevus of Ota, and this fact suggests that clearance of epidermal pigmentation before QSR treatment is important in order to promote the efficiency of the QSR laser for dermal melanocytosis and to reduce PIH induced by inflammation around the basal layer. ${ }^{12}$ Topical bleaching treatment clears postinflammatory hyperpigmentation induced by the QSR laser and also plays an important role as a pretreatment for the next QSR irradiation.

Melasma and ADM are sometimes difficult to distinguish from each other because they are both symmetrical, and they coexist in some cases. Indeed, we have a few cases in our series which were first diagnosed as melasma, but dermal pigmentation was found after the first topical bleaching and the diagnosis was corrected to ADM 
later. In our repeated protocols the topical bleaching treatment can be started for either condition, so the treatment plan can be corrected without any loss of treatment periods.

We here propose repeated treatment protocols for melasma and ADM with better effectiveness than conventional ones, and they may be applied to other pigmented conditions. It may be better that epidermal and dermal pigmentations are treated separately, especially in dark-skinned people who are more likely to suffer PIH after inflammation-inducing therapies.

\section{References}

1. Grimes PE. Melasma. Etiologic and therapeutic considerations. Arch Dermatol 1995;131:1453-7.

2. Hurley ME, Guevara IL, Gonzales M, Pandya AG. Efficacy of glycolic acid peels in the treatment of melasma. Arch Dermatol 2002;138:1578-82.

3. Griffiths CE, Finkel LJ, Ditre CM, Hamilton TA, Ellis CN, Voorhees JJ. Topical tretinoin (retinoic acid) improves melasma. A vehicle-controlled, clinical trial. Br J Dermatol 1993;129:415-21.

4. Nanda S, Grover C, Reddy BS. Efficacy of hydroquinone $(2 \%)$ versus tretinoin $(0.025 \%)$ as adjunct topical agents for chemical peeling in patients of melasma. Dermatol Surg 2004;30:385-8.

5. Sarkar R, Bhalla M, Kanwar AJ. A comparative study of $20 \%$ azelaic acid cream monotherapy versus a sequential therapy in the treatment of melasma in dark-skinned patients. Dermatology 2002;205:249-54.

6. Lawrence N, Cox SE, Brody HJ. Treatment of melasma with Jessner's solution versus glycolic acid: a comparison of clinical efficacy and evaluation of the predictive ability of Wood's light examination. J Am Acad Dermatol 1997;36:589-93.

7. Garcia A, Fulton JE Jr. The combination of glycolic acid and hydroquinone or kojic acid for the treatment of melasma and related conditions. Dermatol Surg 1996;22:443-7.

8. Hori Y, Kawashima M, Oohara K, Kukita A. Acquired, bilateral nevus of Ota-like macules. J Am Acad Dermatol 1984;10:961-4.

9. Yoshimura K, Harii K, Aoyama T, et al. A new bleaching protocol for hyperpigmented skin lesions with a high concentration of all-trans retinoic acid aqueous gel. Aesthetic Plast Surg 1999;23:285-91.

10. Yoshimura K, Harii K, Aoyama T, Iga T. Experience with a strong bleaching treatment for skin hyperpigmentation in
Orientals. Plast Reconstr Surg 2000;105:1097-108.

11. Yoshimura K, Momosawa A, Watanabe A, et al. Cosmetic color improvement of the nipple-areola complex by optimal use of tretinoin and hydroquinone. Dermatol Surg 2002;28:1153-8.

12. Momosawa A, Yoshimura K, Uchida G, et al. Combined therapy using Qswitched ruby laser and bleaching treatment with tretinoin and hydroquinone for acquired dermal melanocytosis. Dermatol Surg 2003;29:1001-7.

13. Yoshimura K, Momosawa A, Aiba E, et al. Clinical trial of bleaching treatment with $10 \%$ all-trans retinol gel. Dermatol Surg 2003;29:155-60.

14. Muindi J, Frankel SR, Miller WH Jr, et al. Continuous treatment with all-trans retinoic acid causes a progressive reduction in plasma drug concentrations: implications for relapse and retinoid "resistance" in patients with acute promyelocytic leukemia. Blood 1992;79:299-303.

15. Regazzi MB, Iacona I, Gervasutti C, Lazzarino M, Toma S. Clinical pharmacokinetics of tretinoin. Clin Pharmacokinet 1997;32:382-402.

Address correspondence and reprint requests to: Kotaro Yoshimura, MD, Department of Plastic Surgery, University of Tokyo School of Medicine, 7-3-1, Hongo, Bunkyo-Ku, Tokyo 113-8655, Japan, or e-mail: yoshimura@cosmetic-medicine.jp. 